\title{
Gravitational Waves from an Axi-symmetric Source in the Nonsymmetric Gravitational Theory
}

\author{
N. J. Cornish, J. W. Moffat and D. C. Tatarski \\ Department of Physics, University of Toronto \\ Toronto, Ontario M5S 1A7, Canada
}

\begin{abstract}
We examine gravitational waves in an isolated axi-symmetric reflexion symmetric NGT system. The structure of the vacuum field equations is analyzed and the exact solutions for the field variables in the metric tensor are found in the form of expansions in powers of a radial coordinate. We find that in the NGT axially symmetric case the mass of the system remains constant only if the system is static (as it necessarily is in the case of spherical symmetry). If the system radiates, then the mass decreases monotonically and the energy flux associated with waves is positive.
\end{abstract}

04.30.+x, 04.50.+h 


\section{INTRODUCTION}

The present work examines gravitational radiation in the Nonsymmetric Gravitational Theory (NGT) (for a recent detailed review see [1]). We probe the NGT asymptotic behaviour in the wave zone using an exact solution. A complementary analysis based on a DeWitt style background field expansion has already been published, announcing the main result of this current work [2].

The motivation for this work is twofold. Firstly, the nature of gravitational radiation is an important physical question which must be addressed in any candidate gravitational theory. Secondly, the literature already contains several incorrect treatments of gravitational radiation in NGT [3 5], and since more incorrect treatments are currently being published [6], it is important that the record be set straight. The reasons why erroneous results were arrived at by these other authors are explained in [7].

In General Relativity (GR) gravitational radiation from bounded sources has been studied not only through the linearized theory but also with the use of exact solutions. The latter was done for the general case of a bounded source in asymptotically flat spacetime [B]. It was found that confining the arguments to the axially symmetric case did not cause any essential loss of generality. Since even the relevant GR calculations are very tedious and the level of technical difficulty in the case of NGT increases considerably, we limit ourselves to the axi-symmetric case. The GR gravitational waves from isolated axially symmetric reflexion symmetric systems were studied in detail in [9]. Since our treatment of the axi-symmetric NGT case is rather parallel, familiarity with this analysis is strongly recommended.

Since NGT was introduced [10] there have been few analytic solutions of the field equations published. The exact solutions known to date include the spherically symmetric vacuum case [11], the spherically symmetric interior case [12,13] and Bianchi type I cosmological solutions with and without matter [14,15]. This, at least in part, follows from the fact that deriving NGT field equations relevant for particular cases of interest is not as technically simple as may be suggested by its superficial similarity to the corresponding GR situations. Firstly, since the underlying geometry is non-Riemannian, neither the fundamental metric tensor $g_{\mu \nu}$ nor the affine connection is symmetric. This does not constitute a serious problem for the choice of the form of $g_{\mu \nu}$, since we can always assume that its nonsymmetric part takes on the isometries of the symmetric part, which in turn has a well defined GR limit. On the other hand, calculating the connection coefficients proves to be a tedious and time consuming exercise, independent of the method chosen. Secondly, the resultant formulae for the nonsymmetric connection coefficients are extremely complicated for all but the simplest forms of the metric, thus becoming unwieldy to use in the derivation of still more complicated field equations.

The NGT quantities presented in this paper were derived with the use of symbolic algebraic computation procedures. To this end, we have used the symbolic computation system Maple.

In Section [1], we briefly summarize the necessary fundamentals of NGT. Section III deals with the coordinate system and generalization of the GR metric to the NGT case. Then in Section IV, we expand the metric in negative powers of a suitably chosen radial coordinate and analyze the field equations. The closing section contains our conclusions.

Throughout this paper we use units in which $G=c=1$. 


\section{NGT VACUUM FIELD EQUATIONS}

The NGT Lagrangian without sources takes the form:

$$
\mathcal{L}=\sqrt{-g} g^{\mu \nu} R_{\mu \nu}(W)
$$

with $g$ the determinant of $g_{\mu \nu}$. The NGT Ricci tensor is defined as:

$$
\begin{array}{r}
R_{\mu \nu}(W)=W_{\mu \nu, \beta}^{\beta}-\frac{1}{2}\left(W_{\mu \beta, \nu}^{\beta}+W_{\nu \beta, \mu}^{\beta}\right) \\
-W_{\alpha \nu}^{\beta} W_{\mu \beta}^{\alpha}+W_{\alpha \beta}^{\beta} W_{\mu \nu}^{\alpha},
\end{array}
$$

and $W_{\mu \nu}^{\lambda}$ is an unconstrained nonsymmetric connection :

$$
W_{\mu \nu}^{\lambda}=W_{(\mu \nu)}^{\lambda}+W_{[\mu \nu]}^{\lambda} .
$$

(Throughout this paper parentheses and square brackets enclosing indices stand for symmetrization and antisymmetrization, respectively.) The contravariant nonsymmetric tensor $g^{\mu \nu}$ is defined in terms of the equation:

$$
g^{\mu \nu} g_{\sigma \nu}=g^{\nu \mu} g_{\nu \sigma}=\delta_{\sigma}^{\mu} .
$$

If we define the torsion vector as:

$$
W_{\mu} \equiv W_{[\mu \nu]}^{\nu}=\frac{1}{2}\left(W_{\mu \nu}^{\nu}-W_{\nu \mu}^{\nu}\right),
$$

then the connection:

$$
\Gamma_{\mu \nu}^{\lambda}=W_{\mu \nu}^{\lambda}+\frac{2}{3} \delta_{\mu}^{\lambda} W_{\nu}
$$

is torsion free:

$$
\Gamma_{\mu} \equiv \Gamma_{[\mu \alpha]}^{\alpha}=0
$$

Defining now:

$$
\begin{gathered}
R_{\mu \nu}(\Gamma)=\Gamma_{\mu \nu, \beta}^{\beta}-\frac{1}{2}\left(\Gamma_{(\mu \beta), \nu}^{\beta}+\Gamma_{(\nu \beta), \mu}^{\beta}\right) \\
-\Gamma_{\alpha \nu}^{\beta} \Gamma_{\mu \beta}^{\alpha}+\Gamma_{(\alpha \beta)}^{\beta} \Gamma_{\mu \nu}^{\alpha},
\end{gathered}
$$

we can write:

$$
R_{\mu \nu}(W)=R_{\mu \nu}(\Gamma)+\frac{2}{3} W_{[\mu, \nu]},
$$

where $W_{[\mu, \nu]}=\frac{1}{2}\left(W_{\mu, \nu}-W_{\nu, \mu}\right)$. Finally, the NGT vacuum field equations can be expressed as:

$$
g_{\mu \nu, \sigma}-g_{\rho \nu} \Gamma_{\mu \sigma}^{\rho}-g_{\mu \rho} \Gamma_{\sigma \nu}^{\rho}=0,
$$




$$
\begin{aligned}
& \left(\sqrt{-g} g^{[\mu \nu]}\right)_{, \nu}=0, \\
& R_{\mu \nu}(\Gamma)=\frac{2}{3} W_{[\nu, \mu]} .
\end{aligned}
$$

For the purpose of the analysis of Section 『V, it is convenient to decompose $R_{\mu \nu}$ into standard symmetric and antisymmetric parts: $R_{(\mu \nu)}, R_{[\mu \nu]}$, and then rewrite the field equation (2.12) in the following form:

$$
\begin{gathered}
R_{(\mu \nu)}(\Gamma)=0, \\
R_{[\mu \nu, \rho]}(\Gamma)=0,
\end{gathered}
$$

where we used equations (2.6), (2.7) and the notation:

$$
R_{[\mu \nu, \rho]}=R_{[\mu \nu]_{, \rho}}+R_{[\nu \rho]_{, \mu}}+R_{[\rho \mu]_{, \nu}} .
$$

\section{THE METRIC}

Similarly to GR, the simplest NGT field due to a bounded source would be spherically symmetric. However, the NGT equivalent of Birkhoff's theorem (see e.g. []]) shows that a spherically symmetric gravitational field in an empty space must be static. Hence, no gravitational radiation escapes into empty space from a pulsating spherically symmetric source.

Following [9] we consider the next simplest case: the field which was initially static and spherically symmetric and eventually becomes such, but undergoes an intermediate nonspherical wave emitting period. Also, spacetime is assumed to be axially symmetric and reflexion-symmetric at all times. Because of the complexity of the field equations, we are forced to use the method of expansion to examine the problem. This approach, namely expanding in negative powers of a radial coordinate, was also used in the GR analysis [9] and naturally suits a wave problem.

Due to the physical picture sketched above and to the fact that we are interested in the asymptotic behaviour of the field at null infinity, $\mathcal{I}$, (in an arbitrary direction from our isolated source) polar coordinates $x^{0}=u, \mathbf{x}=(r, \theta, \phi)$ are the natural choice. The "retarded time" $u=t-r$ has the property that the hypersurfaces $u=$ constant are light-like. Detailed discussion of the coordinate systems permissible for investigation of outgoing gravitational waves from isolated systems can be found in [8,9].

The covariant GR metric tensor corresponding to the situation described above is:

$$
g_{\mu \nu}=\left(\begin{array}{cccc}
V r^{-1} e^{2 \beta}-U^{2} r^{2} e^{2 \gamma} & e^{2 \beta} & U r^{2} e^{2 \gamma} & 0 \\
e^{2 \beta} & 0 & 0 & 0 \\
U r^{2} e^{2 \gamma} & 0 & -r^{2} e^{2 \gamma} & 0 \\
0 & 0 & 0 & -r^{2} e^{-2 \gamma} \sin ^{2} \theta
\end{array}\right)
$$


with $U, V, \beta, \gamma$ being functions of $u, r$ and $\theta$ was first given in [16].

For any metric in polar coordinates, form conditions must be imposed in the neighbourhood of the polar axis, $\sin \theta=0$, to ensure regularity. In the case under consideration we have that, as $\sin \theta \rightarrow 0, V, \beta, U / \sin \theta, \gamma / \sin ^{2} \theta$ each is a function of $\cos \theta$ regular at $\cos \theta= \pm 1$.

In order to find the NGT generalization of the metric tensor (3.1) we require that the symmetric part of the NGT metric tensor be formally the same as the GR metric tensor. We then impose the spacetime symmetries of the symmetric metric onto the antisymmetric sector. This is achieved by enforcing $£_{\vec{\xi}_{(i)}} g_{[\mu \nu]}=0$, where the Killing vector field, $\vec{\xi}_{(i)}$, is obtained from $£_{\vec{\xi}_{(i)}} g_{(\mu \nu)}=0$. The solution to this equation for the metric (3.1) yields the single Killing vector field $\vec{\xi}_{(1)}=\xi_{(1)}^{3} \partial_{\phi}=\sin ^{2} \theta \partial_{\phi}$. Imposing $£_{\vec{\xi}_{(1)}} g_{[\mu \nu]}=0$ yields:

$$
\xi_{(1)}^{3} \partial_{\phi} g_{[\mu \nu]}+g_{[\mu 3]} \partial_{\nu} \xi_{(1)}^{3}+g_{[3 \nu]} \partial_{\mu} \xi_{(1)}^{3}=0
$$

This equation gives $\partial_{\phi} g_{[\mu \nu]}=0$, but does not exclude any antisymmetric components. This is markedly different from the static spherically symmetric case where the above procedure excludes four of the six antisymmetric components. Without further simplification, the NGT calculation would involve ten independent functions and ten independent non-linear differential equations. This would constitute a huge increase in complexity from the system of four equations and functions found in the GR case.

To make the problem tractable, we need to determine which antisymmetric functions can be set to zero. To accomplish this we note that the imposition of axi-symmetry splits the antisymmetric field equations (2.11), (2.14) into two sets of three independent equations. (This can be seen directly from the block-diagonal form of the GR metric). The first set explicitly involves the three skew functions $g^{[01]}, g^{[02]}, g^{[12]}$ :

$$
\begin{gathered}
\left(\sqrt{-g} g^{[\mu \nu]}\right)_{, \nu}=0 \quad(\mu=0,1,2), \\
R_{[01,2]}=0 .
\end{gathered}
$$

These four equations are not independent due to the one identity:

$$
\left(\sqrt{-g} g^{[\mu \nu]}\right)_{, \nu, \mu}=0 \quad(\mu, \nu=0,1,2) .
$$

The second set of four equations explicitly involves the three skew functions $g^{[30]}, g^{[31]}, g^{[32]}$ :

$$
\begin{aligned}
\left(\sqrt{-g} g^{[3 \nu]}\right)_{, \nu} & =0, \\
R_{[3 \mu, \nu]} & =0 .
\end{aligned}
$$

These four equations are also not independent due to the one identity

$$
\epsilon^{3 \mu \nu \rho} R_{[3 \mu, \nu], \rho}=0 .
$$

We may now choose to work with either set of three equations and three functions, noting that eliminating one set of three functions simultaneously eliminates the three corresponding 
equations. We choose to work with the first set of functions and equations as they reproduce the usual static spherically symmetric solution when the $u$ and $\theta$ dependence is suppressed. The components that we discard correspond to the magnetic monopole-like solutions of NGT. These components can be discarded without loss of generality as an extension of the background field analysis of Ref. [2] to include all six skew functions does not alter our conclusions [17].

In view of the above, the NGT generalization of the metric tensor (3.1) is:

$$
g_{\mu \nu}=\left(\begin{array}{cccc}
V r^{-1} e^{2 \beta}-U^{2} r^{2} e^{2 \gamma} & e^{2 \beta}+\omega & U r^{2} e^{2 \gamma}+\lambda & 0 \\
e^{2 \beta}-\omega & 0 & \sigma & 0 \\
U r^{2} e^{2 \gamma}-\lambda & -\sigma & -r^{2} e^{2 \gamma} & 0 \\
0 & 0 & 0 & -r^{2} e^{-2 \gamma} \sin ^{2} \theta
\end{array}\right)
$$

where $\omega, \lambda$ and $\sigma$ are functions of $u, r$ and $\theta$. The contravariant metric tensor is given by:

$$
g^{\mu \nu}=\left(\begin{array}{cccc}
-\sigma^{2} e^{-2 \gamma} A & g^{01} & g^{02} & 0 \\
g^{10} & \left(r e^{2 \beta} V-\lambda^{2} e^{-2 \gamma}\right) A & g^{12} & 0 \\
g^{20} & g^{21} & -\left(e^{4 \beta}-\omega^{2}\right) A e^{-2 \gamma} & 0 \\
0 & 0 & 0 & -r^{-2} e^{2 \gamma} \sin ^{-2} \theta
\end{array}\right)
$$

where

$$
\begin{aligned}
A & =\frac{r^{2} \sin ^{2} \theta}{g}, \\
g & =r^{2} \sin ^{2} \theta\left[-r^{2}\left(e^{4 \beta}-(\omega-\sigma U)^{2}\right)+\sigma e^{2 \beta-2 \gamma}\left(2 \lambda-\sigma \frac{V}{r}\right)\right], \\
g^{01} & =\left[r^{2}\left(\omega-\sigma U-e^{2 \beta}\right)+\sigma \lambda e^{-2 \gamma}\right] A, \\
g^{02} & =\left(e^{2 \beta}-\omega\right) \sigma e^{-2 \gamma} A, \\
g^{12} & =\left[U r^{2}\left(\sigma-\omega-e^{2 \beta}\right)+e^{2 \beta-2 \gamma}\left(\lambda-\sigma V r^{-1}\right)+\lambda \omega e^{-2 \gamma}\right] A, \\
g^{\mu \nu} & =g^{\nu \mu}[(\omega, \sigma, \lambda) \rightarrow(-\omega,-\sigma,-\lambda)] .
\end{aligned}
$$

\section{THE FIELD EQUATIONS}

The analysis determining the form of the functions $U, V, \beta, \gamma, \omega, \lambda, \sigma$ in our case is a natural extension of that given in detail by Bondi et. al. [9] for finding the forms of $U, V, \beta, \gamma$. The requirement that the field contain only outgoing radiation at large distances from the source gives the form of $\gamma$ :

$$
\gamma=\frac{f(u, \theta)}{r}+\frac{g(u, \theta)}{r^{3}}+\ldots
$$

Demanding that the solution have the correct static limit (or equilibrium configuration) leads to the following forms for $U, V, \beta$ and $\gamma$ (unless otherwise stated, all coefficients in the general expansions are functions of both $u$ and $\theta)$ : 


$$
\begin{aligned}
& U=\frac{U_{1}}{r}+\frac{U_{2}}{r^{2}}+\ldots, \\
& V=r-2 M+\frac{V_{1}}{r}+\ldots, \\
& \beta=\frac{B_{1}}{r}+\frac{B_{2}}{r^{2}}+\ldots, \\
& \gamma=\frac{c}{r}+\frac{C-\frac{1}{6} c^{3}}{r^{3}}+\ldots,
\end{aligned}
$$

The skew functions, $\omega, \lambda$ and $\sigma$, are constrained by the requirement that the spacetime is asymptotically Lorentzian and admits inhomogeneous orthochronous Lorentz transformations [8]. This requirement demands that $g_{[\mu \nu]} g^{[\mu \nu]} \rightarrow 0$ as $r \rightarrow \infty$. In our present coordinates this condition is satisfied if $\omega, \lambda$ and $\sigma$ have the following forms:

$$
\begin{aligned}
& \omega=\frac{W_{1}}{r}+\frac{W_{2}}{r^{2}}+\ldots, \\
& \lambda=L_{0}+\frac{L_{1}}{r}+\ldots, \\
& \sigma=S_{0}+\frac{S_{1}}{r}+\ldots
\end{aligned}
$$

The functions $M, c, C, L_{0}$, and $W_{2}$ are all functions of integration. Bondi et. al. refer to $c$ as the "news function" as it controls the form of the gravitational radiation in the symmetric sector. In an analogous way, $L_{0}$ is the "news function" for the antisymmetric sector. Consistent with these identifications, we shall see that the solution reduces to the static, non-radiative case when both $c$ and $L_{0}$ are set to zero. The static limit tells us that only $M$ and $W_{2}$ can be non-zero when the system passes through its equilibrium position and these coefficients will be identified as the mass and NGT charge of the body, respectively.

We begin our analysis of the field equations by considering the simplest set of field equations - the skew divergence equations $\left(\sqrt{-g} g^{[\mu \nu]}\right)_{, \nu}=0$. The $\theta$ component of this set becomes:

$$
0=S_{0, u}-\frac{2\left(S_{0} c\right)_{, u}-S_{1, u}}{r}+\ldots
$$

and since $\sigma$ must equal zero when passing through equilibrium, $S_{0}=S_{1}=0$ always. Inserting this information into the $u$ component directly returns $W_{1}=0$ from the lowest order term. The remaining, $r$, component yields:

$$
0=L_{0} \cot \theta+L_{0, \theta}-W_{2, u}+\ldots
$$

At this stage of the calculation, it is not profitable to continue to work with the skew divergence equations, as the next orders also contain unknown coefficients from the symmetric functions. Somewhat surprisingly, however, we are already in a position to calculate the NGT charge of the body, and to prove that it is conserved. The NGT charge, $l^{2}$, is defined by the Gaussian surface integral

$$
l^{2} \equiv \frac{1}{4 \pi} \int\left(\sqrt{-g} g^{[0 \nu]}\right)_{, \nu} d^{3} x=\frac{1}{2} \int_{0}^{\pi} W_{2} \sin \theta d \theta=<W_{2}>
$$


where the brackets $<>$ denote the angular average. The charge is conserved since

$$
l_{, u}^{2}=\frac{1}{2} \int_{0}^{\pi} W_{2, u} \sin \theta d \theta=\frac{1}{2} \int_{0}^{\pi}\left(L_{0} \sin \theta\right)_{, \theta} d \theta=0 .
$$

This follows from the fact that $L_{0}$ must be regular on the polar axis.

We now turn our attention to the set of field equations $R_{(\mu \nu)}=0$. The affine connections that we require to construct these generalized Ricci tensor components are obtained by solving the system of 64 equations (2.10). The closed form expressions for the non-zero connection components are extremely lengthy. For brevity, we only display the connections expanded in inverse powers of $r$, and only to the order required for our present analysis. The list of expanded non-zero components is given in the appendix.

Rather than provide an exhaustive list of the $R_{(\mu \nu)}=0$ equations, we shall simply exhibit the components to the order that we require to obtain the solution. We begin with the $(r r)$ component which demands:

$$
0=\frac{2 B_{1}}{r^{3}}+\frac{c^{2}+4 B_{2}}{r^{4}}+\frac{6 B_{3}}{r^{5}}+\ldots,
$$

this gives $B_{1}=B_{3}=0$ and $B_{2}=-c^{2} / 4$. The $(r \theta)$ component then gives

$$
0=\frac{U_{1}}{r}+\frac{U_{2}+U_{1} c+c_{, \theta}+2 c \cot \theta}{r^{2}}+\ldots,
$$

which yields $U_{1}=0$ and $U_{2}=-\left(c_{, \theta}+2 c \cot \theta\right)$. Inserting these expressions into the $(u u)$ component results in the important condition

$$
M_{, u}=-c_{, u}{ }^{2}+\frac{1}{2}\left(c_{, \theta \theta}+3 c_{, \theta} \cot \theta-2 c\right)_{, u} .
$$

We can now use the (ur) components of $R_{(\mu \nu)}=0$ at next order to solve for $V_{1}$, but first we must choose how we wish to write the function of integration contained in $U_{3}$. Following Bondi et. al. we shall write $U_{3}$ as

$$
U_{3}=2 N+3 c c_{, \theta}+4 c^{2} \cot \theta,
$$

where $N$ is the additional function of integration (the reason that $U_{3}$ is written in this way rather than as $U_{3}=\widetilde{N}$, and that the second function of integration in $\gamma$ is written as $C-\frac{1}{6} c^{3}$ rather than as $\widetilde{C}$, is that we can then identify $N$ and $C$ as the dipole and quadrupole moments of the source, respectively). Now the (ur) equation at next order:

$$
\begin{aligned}
0= & \sin ^{2} \theta\left(11 c^{2}-5\left(c_{, \theta}\right)^{2}+4 c^{2} c_{, u}-6 c c_{, \theta \theta}\right) \\
& +U_{3} \sin \theta \cos \theta-8 c^{2}-19 c c_{, \theta} \cos \theta \sin \theta \\
& +\sin ^{2} \theta\left(U_{3, \theta}-2 L_{0}^{2}+2 V_{1}\right),
\end{aligned}
$$

can be solved to give

$$
\begin{aligned}
V_{1}= & -N_{, \theta}-N \cot \theta+c_{, \theta}^{2}+4 c c_{, \theta} \cot \theta \\
& +\frac{1}{2} c^{2}\left(1+8 \cot ^{2} \theta\right)+L_{0}^{2} .
\end{aligned}
$$


It is interesting to note that $V_{1}$ contains the first explicit difference between the symmetric functions found for GR and those found for NGT. Substituting the above results into the $(\theta \theta)$ and $(u \theta)$ components of $R_{(\mu \nu)}=0$ produces the following auxiliary conditions on the multipole moments $C, N$ :

$$
\begin{aligned}
4 C_{, u} & =2 c^{2} c_{, u}+2 c M+N \cot \theta-N_{, \theta}-L_{0}^{2}, \\
-3 N_{, u} & =M_{, \theta}+3 c c_{, u \theta}+4 c c_{, u} \cot \theta+c_{, u} c_{, \theta} .
\end{aligned}
$$

We see that the quadrupole moment of a source will decrease more rapidly in NGT than in GR due to the $L_{0}^{2}$ term.

For completeness, we shall now return to the antisymmetric sector where the skew divergence equation can now be used to obtain the additional relations:

$$
\begin{aligned}
W_{3} & =2 S_{2, \theta}+S_{2} \cot \theta, \\
S_{2, u} & =L_{1}-2 c L_{0} .
\end{aligned}
$$

The only remaining antisymmetric field equation (2.14):

$$
R_{[01,2]}=R_{[01], 2}+R_{[12], 0}+R_{[20], 1}=0,
$$

gives to lowest order:

$$
\left(W_{2, \theta}+L_{1}+S_{2, u}\right)_{, u}=0 .
$$

This equation yields the additional relation:

$$
W_{2, \theta}=2 c L_{0}-2 L_{1}
$$

\section{Analysis of the solution}

To demonstrate the physical interpretation of $M$, we consider the static limit. We can scale the function $c$ for either one of the static periods to be $c=0$ (forsaking the $\theta$ dependence of $c$ limits us here to a static spherically symmetric system). We now remove the terms

containing the functions $N$ and $C$ since they correspond to multipole moments. Since there is no radiation during the static period, $N=C=0$. The metric (3.7) tends now to its static spherically symmetric limit:

$$
\begin{aligned}
g_{00} & =1-\frac{2 M_{s}}{r}+\frac{l_{s}^{4}}{r^{4}}-\frac{2 M_{s} l_{s}^{4}}{r^{5}}, \\
g_{(01)} & =1+\frac{l_{s}^{4}}{2 r^{4}} \\
g_{[01]} & =\frac{l_{s}^{2}}{r^{2}} \\
g_{(02)} & =g_{[02]}=0 \\
g_{22} & =-r^{2} \\
g_{33} & =-r^{2} \sin ^{2} \theta
\end{aligned}
$$


where by $M_{s}, l_{s}^{2}$ we denote the static limit of $M$ and $l^{2}$, respectively.

Now a coordinate transformation from the retarded time $u$ to the usual time coordinate $t=u+r$ converts the above metric into the NGT static spherically symmetric metric [1,11]:

$$
\begin{aligned}
d s^{2}= & \left(1+\frac{l_{s}^{4}}{r^{4}}\right)\left(1-\frac{2 M_{s}}{r}\right) d t^{2}-\left(1-\frac{2 M_{s}}{r}\right)^{-1} d r^{2} \\
& -r^{2}\left(d \theta^{2}+\sin ^{2} \theta d \phi^{2}\right),
\end{aligned}
$$

and

$$
g_{[01]}=\frac{l_{s}^{2}}{r^{2}}
$$

Thus, the static spherically symmetric limit, $M_{s}$, of the "mass aspect" $M(u, \theta)$ can only be interpreted as the mass of the system. Similarly, the static spherically symmetric limit, $l_{s}^{2}$, of the "charge aspect" $W_{2}(u, \theta)$ is identically the NGT charge of the system as shown by equations (4.10) and (4.11).

If we define the mass $m(u)$ of the system as the mean value of $M(u, \theta)$ over the sphere:

$$
m(u)=\frac{1}{2} \int_{0}^{\pi} M(u, \theta) \sin \theta d \theta=<M(u, \theta)>
$$

then $c(u, \theta)$ completely determines the time evolution of the mass $m(u)$. Integrating (4.14) and noticing that the second term does not contribute to the integral due to the condition that $c$ be regular on the polar axis, we get

$$
m_{, u}=\frac{d m}{d u}=-\frac{1}{2} \int_{0}^{\pi} c_{, u}^{2} \sin \theta d \theta .
$$

Since we discussed here systems whose initial and final states are static, the physical interpretation of $m(u)$ as the mass of the system is unambiguous. Analogously to the GR case the main result is as follows:

The mass of an axially symmetric NGT system is constant only if the system remains static. If the system evolves in time (emits waves), the mass decreases monotonically.

Since radiation is the only energy loss mechanism available to the system, the above proves that gravitational waves emitted by an axi-symmetric reflexion symmetric NGT source compatible with the metric (3.7) carry positive energy or, in other words, the flux of gravitational energy in NGT is positive.

\section{CONCLUSIONS}

We have proved that an NGT axi-symmetric system emitting gravitational waves has the usual GR-like asymptotic behaviour in the wave zone. The NGT contributions to the physical quantities decay rapidly with the distance from the source and the energy flux at spatial infinity is necessarily positive.

While we concentrated on an axi-symmetric source to simplify computations, the validity of our result is not confined to this particular symmetry. This contention can be made 
concrete by adapting the analysis made by Sachs [8], where it was shown that the axisymmetric solution contains all the important features of any isolated, radiative system in GR. Sachs' result follows from considerations of the asymptotic nature of the spacetime, and these considerations are unchanged in NGT. Physically, we can argue that an axisymmetric source provides a complete range of multipole moments to act as a source of gravitational waves, and thus provides a general test of the wave sector of any gravitational theory. Moreover, in the wave zone, the superposition principle may be used to construct the radiation pattern of any isolated body from a suitable sum of axi-symmetric solutions. Our result totally refutes the recent claims that NGT has bad wave behaviour [4, 6], and shows that aesthetically unappealing, phenomenological modifications to NGT [5] are not necessary.

\section{ACKNOWLEDGEMENTS}

This work was supported by the Natural Sciences and Engineering Research Council of Canada. One of the authors (NJC) is grateful for the support provided by a Canadian Commonwealth Scholarship. We thank P. Savaria for helpful discussions.

$$
\begin{aligned}
\text { APPENDIX: } & \\
\Gamma_{00}^{0}= & 2 B_{1, u} r^{-1}-\left(U_{1} U_{2}+U_{1}^{2} c+M-2 B_{2, u}-B_{1}\right) r^{-2} \\
+ & {\left[2 B_{2}-2 M B_{1}+V_{1}-2 L_{0}^{2}-U_{2}^{2}+2 B_{3, \theta}\right.} \\
& \left.+2 U_{1}\left(U_{2} B_{1}-U_{3}-2 c U_{2}\right)+2 c U_{1}^{2}\left(B_{1}-c\right)\right] r^{-3}+\ldots, \\
\Gamma_{(01)}^{0}= & \left(S_{2} L_{0}+2 W_{2}^{2}\right) r^{-5}+\ldots, \\
\Gamma_{[01]}^{0}=- & 2 W_{2} r^{-3}-\left(3 W_{3}+\frac{1}{2} S_{2} U_{1}-6 B_{1} W_{2}\right) r^{-4}+\ldots, \\
\Gamma_{(02)}^{0}=- & \frac{1}{2} U_{1}+\left(B_{1} U_{1}+B_{1, \theta}\right) r^{-1} \\
& +\left[c U_{2}+B_{2, \theta}+\frac{1}{2} U_{3}+U_{1}\left(B_{2}-B_{1}^{2}+c^{2}\right)\right] r^{-2}+\ldots, \\
\Gamma_{[02]}^{0}= & L_{0} r^{-1}+\left[L_{0}\left(c+2 B_{1}\right)+\frac{1}{2}\left(W_{2, \theta}+S_{2, u}+U_{1} W_{2}-3 L_{1}\right)\right] r^{-2}+\ldots, \\
\Gamma_{(12)}^{0}= & 2 W_{2} S_{2} r^{-5}+\ldots, \\
\Gamma_{[12]}^{0}= & 3 S_{2} r^{-3}+S_{2}\left(8 B_{1}+c\right) r^{-4} \ldots, \\
\Gamma_{22}^{0}= & c+r-2 B_{1}-2\left(B_{2}+c B_{1}-B_{1}^{2}\right) r^{-1} \\
+ & {\left[2 c B_{1}^{2}+4 B_{2} B_{1}-2 B_{2} c-C-\frac{1}{2} c^{3}-2 B_{3}-\frac{4}{3} B_{1}^{3}\right] r^{-2}+\ldots, } \\
\Gamma_{33}^{0} / \sin ^{2} \theta= & r-c-2 B_{1}+2\left(c B_{1}-B_{2}+B_{1}^{2}\right) r^{-1} \\
+ & {\left[4 B_{2} B_{1}-2 c B_{1}^{2}+2 B_{2} c+C+\frac{1}{2} c^{3}-2 B_{3}-\frac{4}{3} B_{1}^{3}\right] r^{-2}+\ldots, } \\
&
\end{aligned}
$$




$$
\begin{aligned}
& \Gamma_{00}^{1}=\left[U_{1}^{2}\left(U_{1, \theta}+c_{, u}\right)-B_{1, u}-M_{, u}\right] r^{-1} \\
& +\left[U_{1}^{2}\left(2\left(c-B_{1}\right)\left(c_{, u}+U_{1, \theta}\right)+U_{2, \theta}+c+c_{, \theta} U_{1}\right)+\frac{1}{2} V_{1, u}-B_{2, u}-B_{1}\right. \\
& \left.+M\left(1+2 B_{1, u}\right)+U_{1} U_{2}\left(1+2 c_{, u}+2 U_{1, \theta}\right)+U_{1}\left(M-B_{1}\right)_{, \theta}\right] r^{-2}+\ldots, \\
& \Gamma_{(01)}^{1}=\frac{1}{2} U_{1}^{2} r^{-1}+\left[U_{1}^{2}\left(c-B_{1}\right)+M-B_{1}+U_{1}\left(\frac{3}{2} U_{2}-B_{1, \theta}\right)\right] r^{-2} \\
& +\left[U_{1}^{2}\left(c^{2}+B_{1}^{2}-B_{2}-2 c B_{1}\right)+3 U_{1} U_{2}\left(c-B_{1}\right)-V_{1}-2 B_{2}\right. \\
& \left.+2 M B_{1}+U_{2}^{2}-U_{1} B_{2, \theta}-U_{2} B_{1, \theta}+2 U_{1} U 3+L_{0}^{2}\right] r^{-3}+\ldots, \\
& \Gamma_{[01]}^{1}=\left(W_{2, u}-\frac{1}{2} L_{0} U_{1}\right) r^{-2} \\
& +\left[W_{2}\left(2-2 B_{1, u}+\frac{1}{2} U_{1}^{2}\right)-L_{0}\left(U_{2}+B_{1, \theta}\right)-2 B_{1} W_{2, u}\right. \\
& \left.+U_{1}\left(\frac{1}{2} W_{2, \theta}-\frac{1}{2} S_{2, u}-L_{1}+L_{0} B_{1}\right)+W_{3, u}-S_{2} U_{1, u}\right] r^{-3}+\ldots, \\
& \Gamma_{(02)}^{1}=\frac{1}{2} U_{1}\left(1-2 U_{1, \theta}-2 c_{, u}\right) \\
& +\left[U_{1}\left(B_{1}\left(2 c_{, u}-1\right)+2 U_{1, \theta}\left(B_{1}-c\right)-U_{2, \theta}-M-2 c c_{, u}\right)\right. \\
& \left.-U_{2}\left(c_{, u}+U_{1, \theta}\right)-M_{, \theta}-U_{1}^{2} c_{, \theta}\right] r^{-1} \\
& +\left[W_{2} L_{0, u}-L_{0} L_{0, \theta}+\frac{1}{2} V_{1, \theta}-U_{3}\left(\frac{1}{2}+U_{1, \theta}+c_{, u}\right)\right. \\
& +U_{2}\left(2 B_{1} c_{, u}-2 c c_{, u}+2 B_{1} U_{1, \theta}-2 c U_{1, \theta}-U_{2, \theta}-c\right) \\
& +U_{1}\left(2 M B_{1}-2 U_{2} c_{, \theta}-\frac{3}{2} c^{2} c_{, u}-c^{2}-B_{2}+B_{1}^{2}-C_{, u}-U_{3, \theta}\right. \\
& +\frac{1}{2} V_{1}-\frac{3}{2} L_{0}^{2}+2\left(B_{1}-c\right)\left(U_{2, \theta}+U_{1} c_{, \theta}\right)-2 c^{2} U_{1, \theta} \\
& \left.\left.+2\left(2 c B_{1}+B_{2}-B_{1}^{2}\right)\left(c_{, u}+U_{1, \theta}\right)\right)\right] r^{-2}+\ldots, \\
& \Gamma_{[02]}^{1}=L_{0, u}+\left[L_{1, u}+L_{0}\left(1-c_{, u}-2 B_{1, u}\right)+L_{0, \theta} U_{1}-2 B_{1} L_{0, u}\right] r^{-1}+\ldots, \\
& \Gamma_{11}^{1}=\quad-2 B_{1} r^{-2}-4 B_{2} r^{-3}-6 B_{3} r^{-4}+2\left(S_{2} L_{0}+2 W_{2}^{2}\right) r^{-5}+\ldots, \\
& \Gamma_{(12)}^{1}=-\frac{1}{2} U_{1}+\left[B_{1, \theta}-U_{2}+U_{1}\left(B_{1}-c\right)\right] r^{-1} \\
& +\left[U_{1}\left(B_{2}-B_{1}{ }^{2}-c^{2}+2 c B_{1}\right)+2 U_{2}\left(B_{1}-c\right)-\frac{3}{2} U_{3}+B_{2, \theta}\right] r^{-2}+\ldots, \\
& \Gamma_{[12]}^{1}=\frac{1}{2}\left(U_{1} W_{2}-L_{1}-W_{2, \theta}+S_{2, u}\right) r^{-2}+\ldots, \\
& \Gamma_{22}^{1}=-r\left(1-c_{, u}-U_{1, \theta}\right) \\
& +\left[2 M+2 c_{, u}\left(c-B_{1}\right)+2 B_{1}\left(1-U_{1, \theta}\right)-c\left(1-2 U_{1, \theta}\right)+U_{2, \theta}+U_{1} c_{, \theta}\right] \\
& +\left[2 B_{2}-2 B_{1}^{2}+2 c B_{1}-4 M B_{1}-V_{1}+2 L_{0}^{2}+2 M c+C_{, u}\right.
\end{aligned}
$$




$$
\begin{aligned}
& +c_{, u}\left(2 B_{1}^{2}-2 B_{2}-4 c B_{1}+\frac{3}{2} c^{2}\right)+c_{, \theta}\left(U_{2}+2 c U_{1}-2 U_{1} B_{1}\right) \\
& \left.+2 U_{1, \theta}\left(c^{2}+B_{1}^{2}-2 c B_{1}-B_{2}\right)+2 U_{2, \theta}\left(c-B_{1}\right)+U_{3, \theta}\right] r^{-1}+\ldots, \\
& \Gamma_{33}^{1} / \sin ^{2} \theta=-r\left(1+c_{, u}-U_{1} \cot \theta\right) \\
& +\left[2 M+c-c_{, \theta} U_{1}+2 c_{, u}\left(c+B_{1}\right)+2 B_{1}-\left(2 U_{1}\left(c+B_{1}\right)-U_{2}\right) \cot \theta\right] \\
& +\left[2 B_{2}-2 B_{1}{ }^{2}-2 c B_{1}-4 M B_{1}-V_{1}-2 M c-C_{, u}\right. \\
& -c_{, u}\left(2 B_{1}^{2}-2 B_{2}+4 c B_{1}+\frac{3}{2} c^{2}\right)-c_{, \theta}\left(U_{2}-2 c U_{1}-2 U_{1} B_{1}\right) \\
& \left.+\left(2 U_{1}\left(c^{2}+B_{1}^{2}+2 c B_{1}-B_{2}\right)-2 U_{2}\left(c+B_{1}\right)+U_{3}\right) \cot \theta\right] r^{-1}+\ldots, \\
& \Gamma_{00}^{2}=-U_{1, u} r^{-1}+\left[U_{1}\left(2 B_{1, u}-2 c_{, u}-U_{1, \theta}\right)-U_{2, u}\right] r^{-2} \\
& +\left[B_{1, \theta}-M_{, \theta}-U_{3, u}-U_{1}^{2}\left(U_{2}+c U_{1}-c_{, \theta}\right)+U_{2}\left(2 B_{1, u}-2 c_{, u}-U_{1, \theta}\right)\right. \\
& \left.+U_{1}\left(B_{1}-U_{2, \theta}+2 B_{2, u}\right)\right] r^{-3}+\ldots, \\
& \Gamma_{(01)}^{2}=-\frac{1}{2} U_{1} r^{-2}+\left(B_{1, \theta}+U_{1} c\right) r^{-3} \\
& +\left[c U_{2}+\frac{1}{2} U_{3}+B_{2, \theta}+2 B_{1, \theta}\left(B_{1}-c\right)\right] r^{-4}+\ldots \\
& \Gamma_{[01]}^{2}=L_{0} r^{-3}-\left[3\left(U_{1} W_{2}+c L_{0}\right)+\frac{1}{2}\left(W_{2, \theta}-S_{2, u}-3 L_{1}\right)\right] r^{-4}+\ldots, \\
& \Gamma_{(02)}^{2}=\left(c_{, u}-\frac{1}{2} U_{1}^{2}\right) r^{-1}+U_{1}\left(B_{1} U_{1}+B_{1, \theta}-\frac{1}{2} U_{2}\right) r^{-2} \\
& +\left[C_{, u}-\frac{1}{2} c^{2} c_{, u}+L_{0}^{2}+U_{1} B_{2, \theta}+U_{2} B_{1, \theta}\right. \\
& \left.+U_{1}^{2}\left(B_{2}+c^{2}-B_{1}^{2}\right)+U_{1} U_{2}\left(B_{1}+c\right)\right] r^{-3}+\ldots, \\
& \Gamma_{[02]}^{2}=-\left(L_{0, \theta}+\frac{1}{2} U_{1} L_{0}\right) r^{-2}+\ldots, \\
& \Gamma_{(11)}^{2}=4 W_{2} S_{2} r^{-7}+\ldots, \\
& \Gamma_{(12)}^{2}=r^{-1}-c r^{-2}+\left(\frac{1}{2} c^{3}-3 C\right) r^{-4}+\ldots, \\
& \Gamma_{[12]}^{2}=-W_{2} r^{-3}+\left(2 W_{2} B_{1}-W_{3}+c W_{2}-\frac{5}{2} S_{2} U_{1}-S_{2, \theta}\right) r^{-4}+\ldots, \\
& \Gamma_{22}^{2}=U_{1}+\left[U_{1}\left(c-2 B_{1}\right)+U_{2}+c_{, \theta}\right] r^{-1} \\
& +\left[U_{3}+U_{2}\left(c-2 B_{1}\right)+2 U_{1}\left(B_{1}^{2}-B_{2}-c B_{1}\right)\right] r^{-2}+\ldots, \\
& \Gamma_{33}^{2} / \sin ^{2} \theta=U_{1}-\cot \theta+\left(U_{2}+c_{, \theta}-c U_{1}-2 B_{1} U_{1}+4 c \cot \theta\right) r^{-1} \\
& +\left[U_{3}-U_{2}\left(c+2 B_{1}\right)+2 U_{1}\left(B_{1}^{2}-B_{2}+c B_{1}\right)\right. \\
& \left.-4 c\left(c_{, \theta}+2 c \cot \theta\right)\right] r^{-2}+\ldots \text {, } \\
& \Gamma_{(03)}^{3}=-c_{, u} r^{-1}+\left(\frac{1}{2} c^{2} c_{, u}-C_{, u}\right) r^{-3}+\ldots, \\
& \Gamma_{[03]}^{3}=L_{0}\left(U_{1}-\cot \theta\right) r^{-2}
\end{aligned}
$$




$$
\begin{aligned}
+ & {\left[W_{2}\left(U_{1} \cot \theta-1-c_{, u}\right)+L_{1}\left(U_{1}-\cot \theta\right)\right.} \\
& \left.+L_{0}\left(c_{, \theta}-2 B_{1} U_{1}+2 c \cot \theta+c U_{1}+U_{2}\right)\right] r^{-3}+\ldots, \\
\Gamma_{(13)}^{3}= & r^{-1}+c r^{-2}+\left(3 C-\frac{1}{2} c^{3}\right) r^{-4}+\ldots, \\
\Gamma_{[13]}^{3}=- & W_{2} r^{-3}+\left[W_{2}\left(2 B_{1}-c\right)+S_{2}\left(U_{1}-\cot \theta\right)-W_{3}\right] r^{-4}+\ldots, \\
\Gamma_{(23)}^{3}= & \cot \theta-c_{, \theta} r^{-1}+\left(\frac{1}{2} c^{2} c_{, \theta}-C_{, \theta}\right) r^{-3}+\ldots, \\
\Gamma_{[23]}^{3}= & -L_{0} r^{-1}+\left[L_{0}\left(2 B_{1}-c\right)-L_{1}\right] r^{-2}+\ldots,
\end{aligned}
$$




\section{REFERENCES}

[1] Moffat J. W. in Gravitation - A Banff Summer Institute, eds. Mann R. B. \& Wesson P., World Scientific, Singapore, 1991, p. 523.

[2] Cornish N. J., Moffat J. W. \& Tatarski D. C., Phys. Lett. A 173, 109, 1993.

[3] Krisher T., Phys. Rev. D 32, 329, 1985.

[4] Damour T., Deser S. \& McCarthy J., Phys. Rev. D 45, R3289, 1992.

[5] Damour T., Deser S. \& McCarthy J., Phys. Rev. D 47, 1541, 1993.

[6] Damour T., To appear in Proceedings of the International Colloquium in Honour of Yvonne Choquet-Bruhat, eds. Flato M., Kerner R. \& Lichnérowicz A., Kluwer, Dordrecht, Netherlands.

[7] Cornish N. J. \& Moffat J. W., Phys. Rev. D 47, 4421, 1993.

[8] Sachs R. K., Proc. Roy. Soc. A, 270, 103, 1962.

[9] Bondi H., van der Burg M. G. J. \& Metzner A. W. K., Proc. Roy. Soc. A, 269, 21, 1962.

[10] Moffat J. W., Phys. Rev. D 19, 3554, 1979.

[11] Moffat J. W., Phys. Rev. D 35, 3733, 1987.

[12] Savaria P., Class. Quantum Grav. 6, 1003, 1989.

[13] Savaria P., Class. Quantum Grav. 9, 1349, 1992.

[14] Kunstatter G., Moffat J. W. \& Savaria P., Phys. Rev. D 19, 3559, 1979.

[15] Kunstatter G., Moffat J. W. \& Savaria P., Can. J. Phys. 58, 729, 1980.

[16] Bondi H., Nature, 186, 535, 1960.

[17] The full static spherically symmetric solution in NGT admits a term $g_{[23]} \sim r^{2} \sin \theta$, which is not compatible with asymptotic inhomogeneous orthochronous Lorentz invariance. It is important to impose such an invariance on the wave solutions so as to facilitate a definition of energy. One may keep the three functions $g_{[03]}, g_{[13]}, g_{[23]}$ in the calculation and maintain asymptotic invariance, if: $g_{[03]}=D_{0}+D_{1} / r+\ldots, g_{[13]}=E_{0}+E_{1} / r+\ldots$, and $g_{[23]}=r F_{-1}+F_{0}+\ldots$, where $D_{i}, E_{i}, F_{i}$ are functions of $u$ and $\theta$. Repeating the calculation in ref. 2 with all six skew functions gives the following: At lowest order the skew divergence equation, $\left(\sqrt{-g} g^{[3 \nu]}\right)_{, \nu}=0$, gives $E_{0, u}=0 \Rightarrow E_{0}=0$. The lowest order in $R_{\{[02], 3]\}}=0$ gives $F_{-1, u u}=0 \Rightarrow F_{-1}=0$ (as demanded by the quasiperiodic boundary conditions). The next order in the skew divergence equations then gives $E_{1, u}=0 \Rightarrow E_{1}=0$. Putting all this into $R_{\{[01], 3\}}=0$ yields $D_{0, u}=0 \Rightarrow D_{0}=0$. Since the two sets of three skew functions have remained uncoupled to this order, the solutions for $g_{[01]}, g_{[02]}, g_{[12]}$ are unchanged from those in ref. 2. At this stage our work is done since all leading terms $(1 / r$ terms in cartesian coordinates) have vanished. It is thus a trivial exercise to show that $g_{[03]}, g_{[13]}, g_{[23]}$ can play no role in determining the flux of gravitational radiation. 